\title{
Shuddhi -A Cleaning Agent
}

\section{Shashank R., Shreyas B., S. Shashank, Yashwanth Venkat Chandolu, Bhavanishankar K.}

\begin{abstract}
Cleaning activities are considered as mundane tasks. These tasks though time-consuming and unpleasant, are essential for a hygienic lifestyle. This issue has been tackled before but they have come at a price, whether in the form of overconsumption of energy resources or prices of such products. Also, they only try to do either dust removal in the form of vacuum cleaning or mopping the floor, but not both. Reducing energy consumption and lowering expenses will help in the widespread usage of such automated alternatives. Combining the two forms of cleaning will help in increasing the versatility of the product. This paper outlines a solution to these issues through the development of a robot. This robot consists of a brush in the front which helps in the removal of dust and a set of mops that cleans the floor using disinfectant water. It can operate in an autonomous mode where it navigates through the room using ultrasonic sensors. It can alternatively be operated manually through the user's smartphone.
\end{abstract}

Keywords: Autonomous Robots, Domestic Help, Manual Control, Mopping, Power efficient, Sweeping

\section{INTRODUCTION}

Eminent chores in our daily life include sanitation which is considered very unpleasant and tiresome. To facilitate this perennial activity, it was necessary to come up with an idea that implements automation. Trends indicate that automation has taken a major leap in the commercial business because of its simplicity and effectiveness.

Sanitation is typically performed using a vacuum cleaner, which draws dust and other particles into a container by the suction created by an electric motor driving a fan. These vacuum cleaners consume a lot of energy resources and are also quite expensive. Therefore, it was necessary to come up with an idea that resolves both of these problems.

Every patent has its pros and cons. They can be efficient or budget-friendly, usually one or the other but not both. This work aspires to render a tangible solution to the predicament of production of an automated cleaner which is efficient while keeping its low cost. The primary mechanism in this robot is provided by the brushes and mops. Not only do they work efficiently but they also will consume fewer resources than the traditional vacuum cleaners.

Revised Manuscript Received on November 21, 2019.

Mr. Shashank R., Department of Computer Science Engineering, RNSIT, Bangalore, India. Email: shashankperso@gmail.com

Mr. Shreyas B., Department of Computer Science Engineering, RNSIT, Bangalore, India. Email: shreyas.badri27@gmail.com

Mr. S. Shashank, Department of Computer Science Engineering, RNSIT, Bangalore, India. Email: samavedulashashank99@gmail.com

Mr. Yashwanth Venkat Chandolu, Department of Computer Science Engineering, RNSIT, Bangalore, India. Email: ychandolu0131@gmail.com

Dr. Bhavanishankar K., Department of Computer Science Engineering, RNSIT, Bangalore, India. Email: Bsharsh@gmail.com
The proposed design will be operated in two modes. In the first mode, the robot is completely independent. It does not require human interference. Decisions are made after processing the data from the ultrasonic sensors and water-level sensors by the microcontroller. In the second mode, the robot can be manipulated into cleaning a specific region of. Eminent chores in our daily life include sanitation which is considered very unpleasant and tiresome. To facilitate this perennial activity, it was necessary to come up with an idea that implements automation. Trends indicate that automation has taken a major leap in the commercial business because of its simplicity and effectiveness.

Sanitation is typically performed using a vacuum cleaner, which draws dust and other particles into a container by the suction created by an electric motor driving a fan. These vacuum cleaners consume a lot of energy resources and are also quite expensive. Therefore, it was necessary to come up with an idea that resolves both of these problems.

Every patent has its pros and cons. They can be efficient or budget-friendly, usually one or the other but not both. This work aspires to render a tangible solution to the predicament of production of an automated cleaner which is efficient while keeping its low cost.

The primary mechanism in this robot is provided by the brushes and mops. Not only do they work efficiently but they also will consume fewer resources than the traditional vacuum cleaners.

The proposed design will be operated in two modes. In the first mode, the robot is completely independent. It does not require human interference. Decisions are made after processing the data from the ultrasonic sensors and water-level sensors by the microcontroller. In the second mode, the robot can be manipulated into cleaning a specific region of the floor by controlling it on a mobile application using Bluetooth.

This robot cleaner will reduce the burden on every household, industry, and warehouse by minimizing their workload and solving the simple but prominent chore. efficiently but they also will consume fewer resources than the traditional vacuum cleaners.

\section{RELATED WORK}

The objective paper that is presented in [1] proposes the design of a fully autonomous vacuum cleaning robot. It operates in both autonomous as well as in manual mode. The subject robot operates in autonomous mode as well as in manual mode. The robot named as CLEAR- (cleaning entresol autonomous robot), it has variable speed and power-efficient.

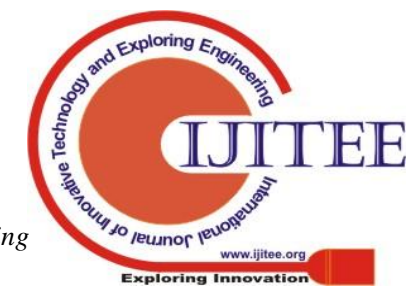




\section{Shuddhi -A Cleaning Agent}

The energy of the robot is saved by the manual mode. Upon completing the path, the robot cleans itself in the station from where it started its cleaning.

An autonomous floor-cleaning robot system presented in [2], which includes a transport drive and control system arranged for autonomous movement of the robot over a floor for performing cleaning operations. The robot has two cleaning zones, one collects loose particulates and the other applies cleaning fluid to scrub the surface and thereafter collects waste liquid from the surface.

An initial design of a dual-purpose cleaning robot that is capable of cleaning both walls and floors overcomes some problems faced by the earlier developed models. It uses an Electric Ducted Fan (EDF) and support ropes from a base module placed on the parapet for adhering the cleaning module to the glass wall, hence performing both dry and wet cleaning. After dry cleaning, the robot performs self-cleaning. This idea was presented in [3].

The disk-shaped robot in [4] equips vacuuming and cleaning controlled by Arduino Mega microcontroller. Obstacle detection and uninterrupted navigation is performed via four ultrasonic sensors, placed 90 degrees apart. The robot not only memorizes this routine but adapts itself to new surroundings and decides accordingly.

Multi-Level planning for cleaning tasks is proposed in [5] which are to be performed by low-cost robotic vacuum cleaners. In general, the robot must find its pose first and only then move to the final destination to cleaning out. The above approach is demonstrated in both simulated and real robots. The results show that even only for localization, the proposed model obtained better results, precisely because the POMDP model itself is capable to deal with the uncertainty of the real world.

A Floor Cleaning Robot which can work in both manual and automatic mode is presented in [6]. All hardware and software operations are controlled by the AT89S52 microcontroller. This robot can perform sweeping and mopping tasks. It can be used in crowded places such as houses, train stations, airports, etc. In the manual mode, the keypad is used to control the robot and to perform the tasks. Small rooms with lesser surface area are tough to clean using this approach.

An approach to build an efficient floor cleaning ROBOT with suction, sweeping and mopping operations is put forth in [7]. This paper presents a method to automatically clean dirty surfaces by using a vacuum cleaner and microcontroller. The optimum path algorithm is coded into the microcontroller which forms the software part. A $12 \mathrm{~V}$ battery supply is used to supply power to all the components. In case the battery level is below the threshold, a buzzer is triggered which cautions the user to charge the battery before using. The battery must be connected to the charger manually and cannot be done automatically.

The literature review resulted in the following caveats. Most of the proposed robots are big which makes it difficult to clean places with smaller surface areas. The robots can either be operated manually or in an automatic mode with no seamless transition between them. Also, none of the robots were able to clean and sweep the floor simultaneously. The above issues are addressed in this paper.

\section{PROPOSED SOLUTION APPROACH}

Development of the robot which takes care of the limitations mentioned above is listed here. The overall working of the proposed approach can be categorized into two components. The first component handles the mobility of the robot and the other monitors the cleaning activity. The pseudo code for the overall working of proposed solution approach is given in the algorithm 1 .

Algorithm 1: Clean_Sweep

Step 1: Start

Step 2: Check if power is sufficient. If yes, proceed to Step 3 else stop after raising alarm.

Step 3: Check if the water level is sufficient. If yes, go to step 4 else raise alarm and stop.

Step 4: Move forward.

Step 5: Check for timeout. If timeout condition satisfies then go back to step 2 else go to Step 4.

Step 6: As it moves forward, check for obstacles or corners of the room. If true, turn around else go to Step 4.

Step 7: The path traversed till it encounters an obstacle I can be stored. The stored data can be used to clean the same room at a faster rate. (yet to be implemented... )

Step 8: Check if the robot has reached the diagonally opposite end of the room. If true, terminate the operation and stop. Else go to Step 4

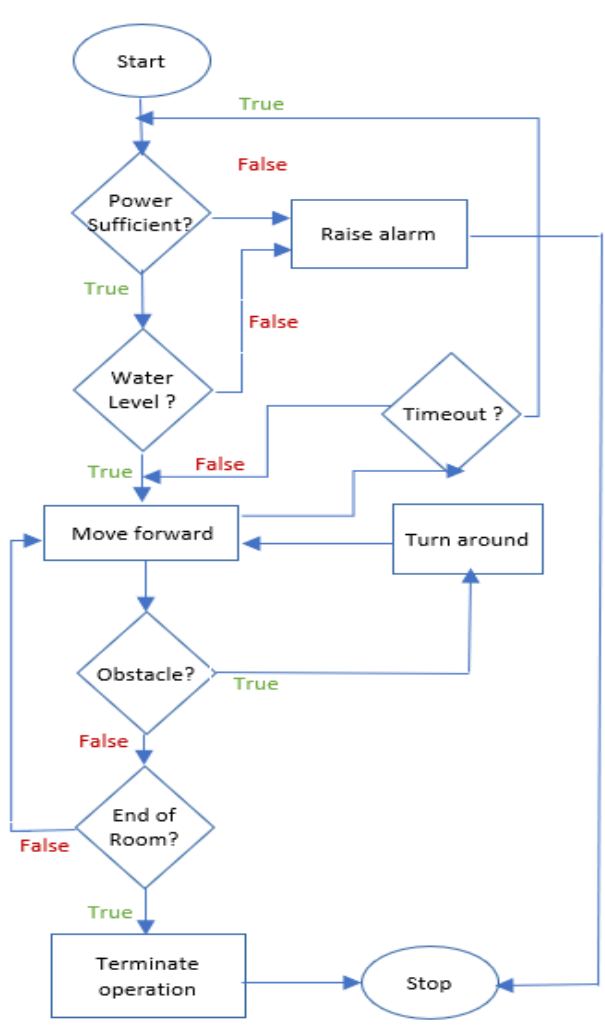

Fig. 1 Automatic Navigation

The flowchart shown in Fig 1 illustrates the movement of the robot and the detailed description is explained in the following paragraph. ence Engineering \& Sciences Publication 


\section{Initial setup:}

This involves series of self-diagnostic activities that includes checking current battery level and water content in the tank. An alarm is raised if either of these falls below a certain threshold. The user can then choose one of the two available modes: automatic or manual. The modes of operation can be selected either by the on-board switch or through the smartphone application.

\section{Automatic Mode:}

Upon activation of this mode of operation, the robot navigates through the cleaning area in a snake-like pattern (shown in Fig 2), i.e. it proceeds in a straight path until it encounters an obstacle. While navigating through the room, the cleaning activities are performed by the robot. Three ultrasonic sensors are used to guide its path away from an obstacle. In case of a wall, the bot makes a 180 degree shift and moves in a parallel path to the original. Diagnostic checks are performed periodically (around 20 seconds) to verify that the battery and water are in adequate quantities. (refer Fig 1)

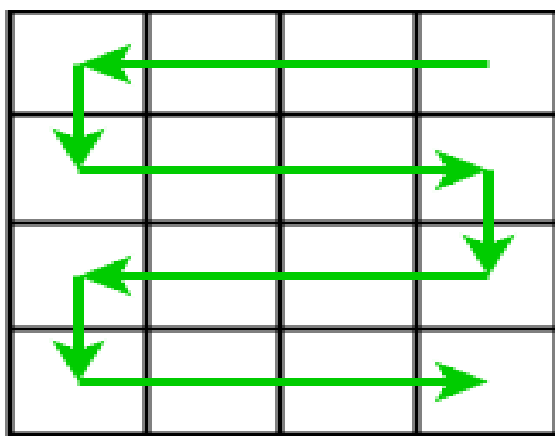

Fig 2 - Snake Traversal

\section{Manual Mode:}

This mode allows the user to take full control of the robot's movements with the help of a smartphone application connected to the robot through Bluetooth. In addition to this, the application facilitates the user to control the cleaning activities of the robot.

\section{Key Components}

The Instrumental Design for the proposed robot is presented in Fig - 3. The main components of the robot are:

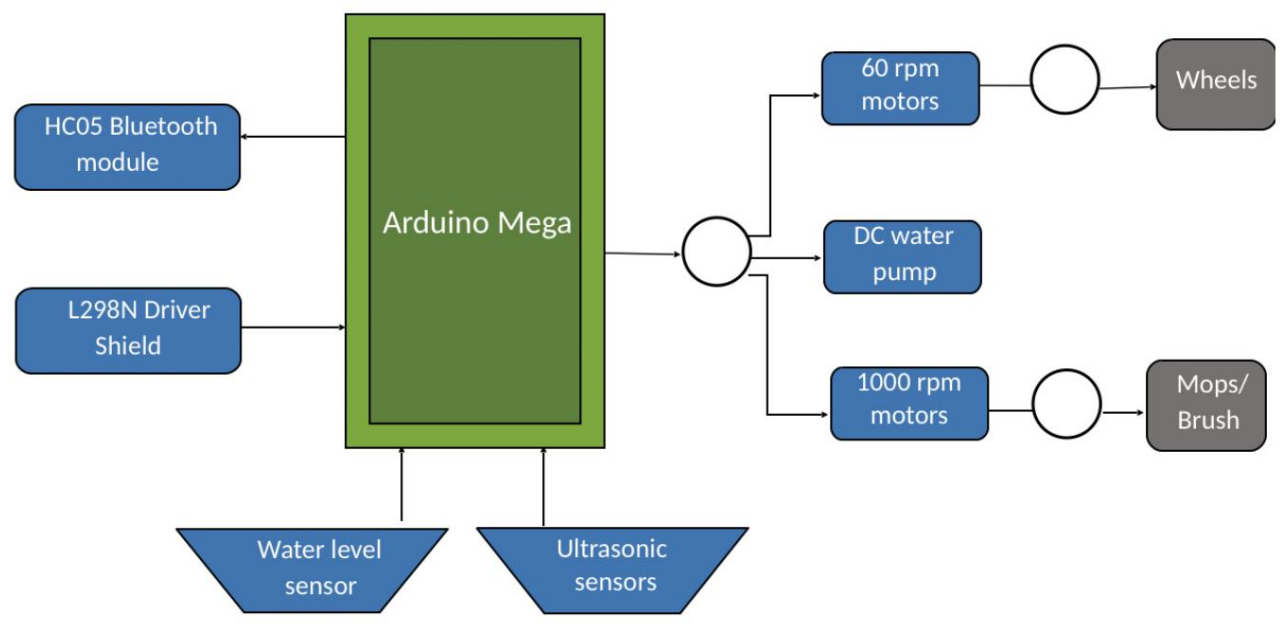

Fig 3 - Block Diagram

\section{Chassis:}

It houses the various immovable components of the robot like the micro-controller (Arduino Mega), batteries, wheel motors etc. These components are covered in a shroud over which the water container is located.

\section{Microcontroller:}

It is the central controlling and processing unit of an embedded system. The proposed microcontroller to be used in this robot is Arduino Mega as it is easy to program and has ample pins for interfacing the various components that are used.

\section{Ultrasonic sensor:}

It acts as the eyes of the robot. It consists of a transmitter and a receiver which emits ultrasonic waves to determine obstacles. On the confirmation of the presence of an obstacle, the robot is then moved in an appropriate direction so as to avoid it.

\section{Bluetooth module:}

It enables the robot to be connected and controlled by the user via a smartphone application. The proposed module is the HC05 as it communicates with the help of USART at 9600 baud rate hence it is easy to interface with any microcontroller that supports USART.

\section{Wheels:}

They enable the robot to move along its path. These are connected to the Arduino through 60 rpm motors. Brush: It is used to sweep the floor. It remains in contact with the floor until a timeout period (say 25 seconds) which allows it to accumulate dust particles in front of it. The robot then stops and the brush is raised and swiveled to sweep the dust inside the dust pan. The rotation of the brush is controlled by the microcontroller through $1000 \mathrm{rpm}$ DC motors fixed on a mechanical arm.

\section{Dust Pan:}

It collects the dust swept by the brush. It is located beneath the robot, just behind the brush. It is not fixed to the robot, but rather it is slid in the rectangular frame. 


\section{Shuddhi -A Cleaning Agent}

It can thus, be removed once the dust collection capacity has reached its maximum.

\section{Water pump motor:}

It allows the microcontroller to control the amount of water that is flowing out of the water tank.

\section{Water tank:}

It holds the hot water which is mixed with the disinfectant that is passed on to the sprinkler. It has a water level sensor which informs the micro controller if the water inside is not sufficient.

\section{Mops:}

They are responsible for the wet wiping operation of the robot. They are circular disks covered in wiping cloth/sponge which wipe the water on the surface.

\section{Driver Shield:} of the robot. The L298N is the recommended driver shield for the robot as it can control up to 4 DC motors, or 2 DC motors with directional and speed control.

\section{Water Level Sensor:}

This is used to measure the amount of water present in the tank. If the water level falls below a threshold level, a trigger is generated which raises an alarm.

The performance of the Robot can be measured through PEAS, whose generalized representation is shown in Fig 4.

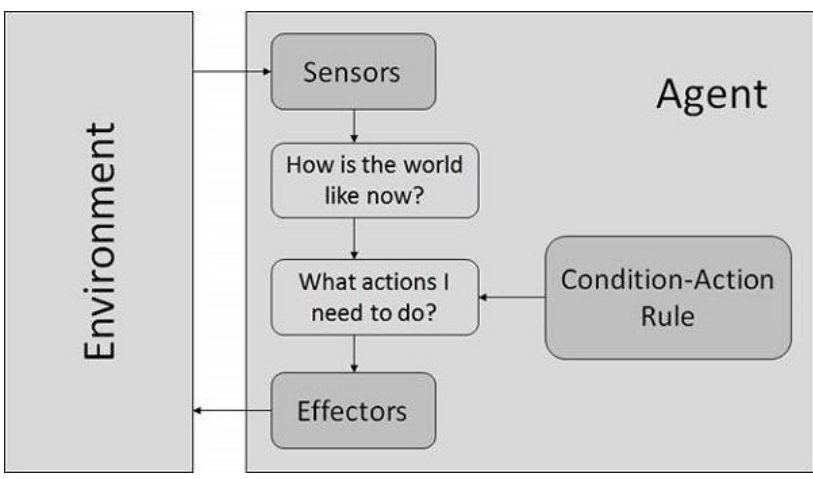

Fig.4 - Representation of PEAS

\section{Performance:}

The performance factors for the autonomous cleaning robot are efficiency in dirt collection and mopping the floor to an appreciable extent.

\section{Environment:}

The typical environments for the robot include rooms, corridors, and halls.

\section{Actuators:}

the parts of the robot which help it to move are the wheels and servo motors cleaning activities are governed by $1000 \mathrm{rpm}$ motors, brush, and mops.

\section{Sensors:}

The sensors used are ultrasonic sensors and water level sensor.

\section{Cleaning Process:}

The cleaning activity of the robot is further divided into two activities that are performed simultaneously: -
The driver shield is used to control the speed and movement

\section{Sweeping activity:}

This section allows the robot to collect dry dust particles from the surface. This is achieved by the use of a brush and a dust pan. The brush is controlled by a mechanical arm present in the front of the robot which sweeps the dust towards the robot. This swept dirt is collected in a dust pan that is present under the robot chassis. This implementation of dust removal consumes lesser power as compared to vacuum cleaners.

\section{Mopping activity:}

This section is responsible for mopping the surface. This section can be broken down into-

- Water dispensing: This is achieved by the water tank and the water pump. A thin tube originating from the bottom of the water tank and terminating at a cavity present in the center of the chassis acts as the outlet from which water is dripped on the surface. The amount of water flowing out is controlled by the microcontroller via the water pump. Water is dropped on the surface in adequate amounts at periodic intervals of time (say 2 seconds).

- Wiping: The components responsible for this are the mops. The mops are fixed at the back of the robot and are controlled by the microcontroller with the help of DC motors. The mops rotate in opposite directions so as to wipe the water on the ground which was dripped by the water dispenser.

\section{CONCLUSION}

The paper proposes the design of an automated floor cleaning robot. The robot can work in either automatic or manual modes. In the automatic mode, the robot performs its job on its own and the manual mode enables the user to control the robot through a smartphone application. In automatic mode, it uses sensors for obstacle detection and moves in a snake-like pattern. The cleaning activities of the robot includes sweeping and mopping. Sweeping is done using the brushes and the dust pan while for mopping the robot utilizes the mops attached to it. The main aim of the development of the robot is to make it autonomous. The navigation path is partially observable as there might be obstacles in the robot's traversal. The path traversed by the robot can be stored and can be used for faster and optimum cleaning process. This can be implemented by using Algorithms, and Machine Learning techniques. The proposed robot aims to make the job of floor cleaning more effective and easier.

\section{REFERENCES}

1. Khalid, Uman, Muhammad Faizan Baloch, Haseeb Haider, M. U. Sardar, M. F. Khan, A. B. Zia, and T. A. K. Qasuria, "Smart floo cleaning robot (clear)", IEEE Standard for User Interface Elements in Power Control of Electronic Devices employed in Office/Consumer Environments (2015).

2. Morse, Christopher John, Andrew Ziegler, Duane Gilbert Jr, and Andrew Jones, "Autonomous surface cleaning robot for dry cleaning", U.S. Patent 7,620,476, issued November 17, 2009.

3. Vishaal, Raj, P. Raghavan, R. Rajesh, Sachin Michael, and Mohan Rajesh Elara, "Design of Dual Purpose Cleaning Robot", Procedia computer science 133 (2018): 518-525. 
4. Asafa, T. B., T. M. Afonja, E. A. Olaniyan, and H. O. Alade "Development of a vacuum cleaner robot", Alexandria engineering journal 57, no. 4 (2018): 2911-2920.

5. Pinheiro, Paulo, Eleri Cardozo, Jacques Wainer, and Eric Rohmer, "Cleaning task planning for an autonomous robot in indoor places with multiples rooms", International Journal of Machine Learning and Computing 5, no. 2 (2015).

6. Kaur, Manreet, and Preeti Abrol, "Design and development of floor cleaner robot (automatic and manual)", International Journal of Computer Applications 97, no. 19 (2014).

7. Thomas, Ajith, M. S. Rohith, Febin Jolly, Jeeson Cheriyan, and R. M. George, "An Advanced Mobile Robot for Floor Cleaning", In National Conference on Recent Advances in Electrical \& Electronics Engineering (NCREEE'16), vol. 3, no. 5, pp. 102-108 (2016).

\section{AUTHORS PROFILE}
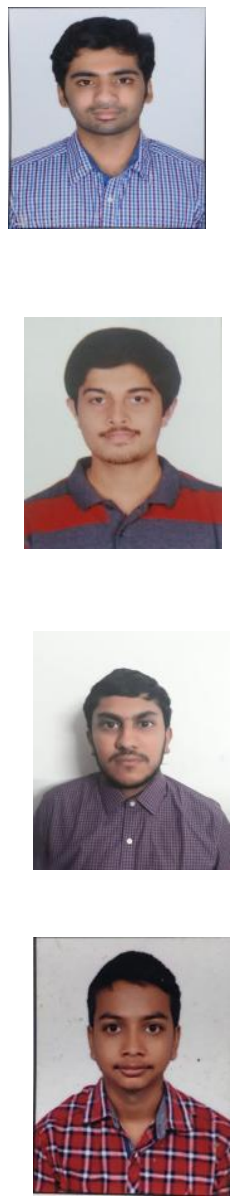

Shashank R., pre-final year B.E. student pursuing Computer Science and Engineering at RNS Institute of Technology, Bengaluru, affiliated to Visveswaraya Technological University, Belagavi. He has presented a paper at Computer Society of India. His areas of interests include Automation and Machine learning.

Shreyas B., is a pre-final year B.E. student pursuing Computer Science and Engineering at RNS Institute of Technology, Bengaluru, affiliated to Visveswaraya Technological University, Belagavi. He has presented a paper at Computer Society of India. His areas of interests include Artificial Intelligence and Machine. Learning.

S. Shashank, is a pre-final year B.E. student pursuing Computer Science and Engineering at RNS Institute of Technology, Bengaluru, affiliated to Visveswaraya Technological University, Belagavi. He has presented a paper at Computer Society of India and at IEEE conference. His areas of interests include Algorithms and Machine Learning.

Yashwanth Venkat Chandolu, is a pre-final year B.E. student pursuing Computer Science and Engineering at RNS Institute of Technology, Bengaluru, affiliated to Visveswaraya Technological University, Belagavi. $\mathrm{He}$ has presented a paper at Computer Society of India. His areas of interests include Algorithms, Database Management and Android App Development.

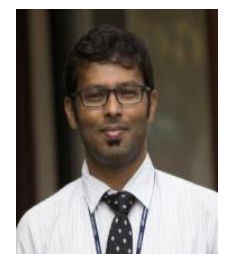

Dr. Bhavanishankar K is an Assistant Professor of Computer Science and Engineering departmen at RNSIT, with an experience of 16 years in teaching and 7 years in research (inclusive). He has published 8 papers in international journals. His area of interests is image processing, algorithms, database management and artificial intelligence. 